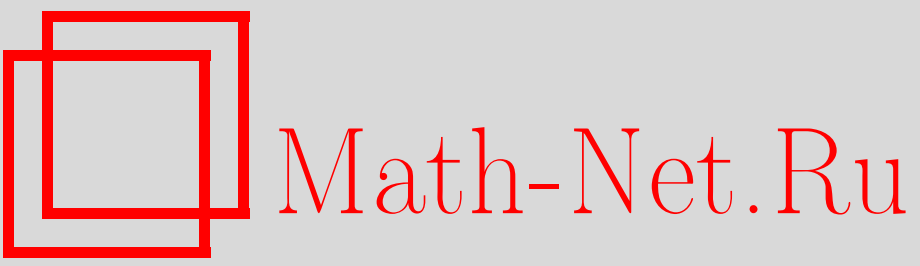

Д. П. Санкович, Метрические свойства боголюбовских траекторий в теории статистического равновесия, ТМФ, 2001, том 127, номер 1, 125-142

DOI: https://doi.org/10.4213/tmf452

Использование Общероссийского математического портала Math-Net.Ru подразумевает, что вы прочитали и согласны с пользовательским соглашением

http://www.mathnet.ru/rus/agreement

Параметры загрузки:

IP: 54.224 .187 .69

26 апреля 2023 г., 18:19:11 
ТЕОРЕТИЧЕСКАЯ

И МАТЕМАТИЧЕСКАЯ

ФИЗИКА

Том 127, № 1

апрель, 2001

(C) 2001 г.

Д. П. Санкович*

\section{МЕТРИЧЕСКИЕ СВОЙСТВА БОГОЛЮБОВСКИХ ТРАЕКТОРИЙ В ТЕОРИИ СТАТИСТИЧЕСКОГО РАВНОВЕСИЯ}

Рассмотрены некоторые свойства меры Боголюбова, возникающей в теории статистического равновесия квантовых систем. Установлена недифференцируемость боголюбовских траекторий в соответствующем функциональном пространстве. Доказана теорема о квадратичной вариации траекторий. Изучены свойства масштабных преобразований, вытекающие из этой теоремы. Построены примеры полугрупп, связанных с мерой Боголюбова. Найдены независимые прирашения для данной меры. Рассмотрена связь меры Боголюбова с параболическими дифференциальными уравнениями в частных производных.

\section{1. СТОХАСТИЧЕСКИЕ ПРОШЕССЫ И МЕРА БОГОЛЮБОВА}

В работе рассмотрены некоторые свойства меры Боголюбова, возникающей в теории статистического равновесия квантовых систем. В частности, важное значение для физических приложений, связанных с построением строгой теории фазовых переходов в неидеальных бозевских системах, имеет исследование структуры носителя этой меры и выяснение связи боголюбовской меры с дифференциальными параболическими уравнениями. Некоторые результаты, полученные в данном направлении, представлены в предлагаемой статье.

При изучении вероятностных мер в функциональных пространствах широко используются понятия и методы теории стохастических процессов. Предположим, что фиксировано некоторое вероятностное пространство $\{\Omega, G, P\}$, где $\Omega$ - пространство элементарных событий $\omega$ с выделенной на нем $\sigma$-алгеброй подмножеств событий $G$ и мерой - вероятностью событий - $P$ на $G$. Числовые функции $f(\omega)$, заданные на $\Omega$ и измеримые относительно $P$, называются случайными величинами. Для интегрируемых относительно меры $P$ функций определяется интеграл (математическое ожидание)

$$
M f(\omega)=\int_{\Omega} f(\omega) d P(\omega)
$$

* Математический институт им. В.А. Стеклова РАН, Москва, Россия 
Случайным элементом со значением в пространстве $X$ называют слабо измеримое отображение $x(\omega)$ пространства $\Omega$ в $X$, т.е. такое отображение, при котором функционал $\langle\xi, x(\omega)\rangle$ измерим относительно меры $P$ для любого $\xi \in X^{\prime}$, где $X^{\prime}$ - сопряженное с $X$ пространство. Если задан случайный элемент $x(\omega)$ со значениями в $X$, то на $\sigma$-алгебре, порожденной цилиндрическими множествами пространства $X$, можно определить вероятностную меру

$$
\mu\left\{x \in X:\left[\left\langle\xi_{1}, x\right\rangle, \ldots,\left\langle\xi_{n}, x\right\rangle\right] \in A_{n}\right\}=P\left\{\omega \in \Omega:\left[\left\langle\xi_{1}, x(\omega)\right\rangle, \ldots,\left\langle\xi_{n}, x(\omega)\right\rangle\right] \in A_{n}\right\},
$$

где $A_{n}$ - любое борелевское множество в $R^{n}$, а векторы $\xi_{1}, \ldots, \xi_{n}(n=1,2, \ldots)$ принадлежат сопряженному пространству $X^{\prime}$. В этом случае

$$
\int_{\Omega} F[x(\omega)] d P(\omega)=\int_{X} F(x) d \mu(x)
$$

для любого функционала $F$, для которого сушествует один из этих интегралов.

Пусть $X$ - некоторое пространство действительных функций аргумента $t \in T$, где $T$ - подмножество $R$. Тогда случайный элемент $x(\omega)$ называют случайной функцией и обозначают $x(\omega, t)$. Аргумент $\omega$ в функции $x(\omega, t)$ часто опускают. Если $t$ интерпретировать как время, то такие случайные функции называют случайными или стохастическими процессами. Случайная функция считается заданной, если заданы ее конечномерные распределения. Случайная функция $x(t)=x(\omega, t)(t \in T)$ со значениями на вероятностном пространстве $\{X, G, P\}$ называется гауссовым процессом, если все ее конечномерные распределения гауссовы. Это означает, что совместное распределение значений $x\left(t_{1}\right), x\left(t_{2}\right), \ldots, x\left(t_{n}\right)$ этого случайного процесса задается функцией плотности

$$
p\left(u_{1}, \ldots, u_{n}\right)=(2 \pi)^{-\frac{n}{2}}(\operatorname{det} B)^{-\frac{1}{2}} \exp \left[-\frac{1}{2} \sum_{i, j=1}^{n} b_{i j}^{(-1)}\left(u_{i}-m\left(t_{i}\right)\right)\left(u_{j}-m\left(t_{j}\right)\right)\right]
$$

где математическое ожидание $m(t)=M[x(\omega, t)]$ и корреляционная функция

$$
B(t, s)=M[(x(\omega, t)-m(t))(x(\omega, s)-m(s))]
$$

$B$ - матрища с элементами $B\left(t_{i}, t_{j}\right)(i, j=1,2, \ldots, n)$, a $b_{i j}^{(-1)}$ - элементы матрицы $B^{-1}$, обратной к матрице $B$. Таким образом, если $X$ является функциональным пространством, то имеет место равенство

$$
\int_{\Omega} F[x(\omega, t)] d P(\omega)=\int_{X} F[x(t)] d \mu(x),
$$

и поэтому задача интегрирования по гауссовой мере в функциональном пространстве эквивалентна задаче интегрирования по мере, порожденной соответствующим случайным гауссовым процессом.

Далее будет регулярно использоваться описанная выше связь теории гауссовых случайных процессов и функционального интегрирования по гауссовым мерам. 
Пусть $\vec{t}=\left(t_{1}, t_{2}, \ldots, t_{n}\right)$ обозначает набор действительных чисел таких, что $0<t_{1}<$ $t_{2}<\cdots<t_{n} \leqslant \beta$. Для заданного произвольного подмножества $E \subset R^{n}$ определим цилиндрическое множество $Q_{\vec{t}}(E)=\left\{x \in X:\left(x\left(t_{1}\right), \ldots, x\left(t_{n}\right)\right) \in E\right\}$. При фиксированном $\vec{t}$ множества $E$ и $Q_{\vec{t}}(E)$ определяют друг друга однозначно. Гауссова центрированная мера заданного цилиндрического множества $Q_{\vec{t}}(E)$ есть по определению

$$
\mu\left\{Q_{\vec{t}}(E)\right\}=(2 \pi)^{-\frac{n}{2}}(\operatorname{det} K)^{-\frac{1}{2}} \int_{E} \exp \left(-\frac{1}{2} \sum_{i, j=1}^{n} k_{i j}^{(-1)} u_{i} u_{j}\right) d u_{1} \ldots d u_{n}
$$

В случае меры Боголюбова [1] пространство $X=C^{\circ}[0, \beta]$, где $C^{\circ}[0, \beta]$ - пространство непрерывных на отрезке $[0, \beta]$ функций с равномерной метрикой

$$
\rho=\max _{t \in[0, \beta]}|x(t)-y(t)|
$$

удовлетворяющих условию $x(0)=x(\beta)$. Билинейный функционал $K(\varphi, \psi)$, определенный на сопряженном пространстве $X^{\prime}$, имеет вид

$$
K(\varphi, \psi)=\int_{0}^{\beta} \int_{0}^{\beta} B(t, s) d \varphi(t) d \psi(s)
$$

где $\varphi(t) \in X^{\prime}=V_{0}[0, \beta]$ - пространство функций ограниченной вариации на $[0, \beta]$, удовлетворяющих условию

$$
\varphi(0)=0, \quad \varphi(t)=\frac{1}{2}[\varphi(t+0)+\varphi(t-0)] \text { при } t \in(0, \beta) .
$$

Матричные элементы матрищы ковариаций имеют вид [2]

$$
k_{i j}=B\left(t_{i}, t_{j}\right)=\frac{1}{2 m \omega \operatorname{sh} \frac{\beta \omega}{2}} \operatorname{ch}\left(\omega\left|t_{i}-t_{j}\right|-\frac{\beta \omega}{2}\right) \text {. }
$$

Мера Боголюбова имеет нулевое среднее.

Рассмотрим некоторые частные случаи формулы (1) для боголюбовской меры.

Пусть $0<t \leqslant \beta$. Вычислим функцию

$$
F_{x(t)} \equiv \mu_{\mathrm{Б}}\{x \in X: x(t) \leqslant \gamma\}
$$

где $\gamma$ - любое действительное число. Используя формулы (1), (2), имеем

$$
\begin{aligned}
F_{x(t)} & =\frac{1}{\sqrt{2 \pi K(\varphi, \varphi)}} \int_{-\infty}^{\gamma} \exp \left(-\frac{1}{2} \frac{u^{2}}{K(\varphi, \varphi)}\right) d u= \\
& =\frac{1}{\sqrt{\frac{\pi}{m \omega} \operatorname{cth} \frac{\beta \omega}{2}}} \int_{-\infty}^{\gamma} \exp \left(-\frac{u^{2}}{2} \frac{2 m \omega}{\operatorname{cth} \frac{\beta \omega}{2}}\right) d u .
\end{aligned}
$$


Из последней формулы видно, что случайная величина $G(x)=x(t)$ нормально распределена с нулевым средним и дисперсией $(2 m \omega)^{-1} \operatorname{cth}(\beta \omega / 2)$.

Пусть $0<t_{1}<t_{2} \leqslant \beta$ и пусть $\gamma-$ любое действительное число. Найдем функцию

$$
F_{x\left(t_{2}\right)-x\left(t_{1}\right)}=\mu_{\mathrm{Б}}\left\{x \in X: x\left(t_{2}\right)-x\left(t_{1}\right) \leqslant \gamma\right\}
$$

Можно записать, что

$$
F_{x\left(t_{2}\right)-x\left(t_{1}\right)}=\mu_{\mathrm{Б}}\left\{x \in X:\left(x\left(t_{1}\right), x\left(t_{2}\right)\right) \in E\right\}
$$

где $E=\left\{\left(u_{1}, u_{2}\right) \in R^{2}: u_{2}-u_{1} \leqslant \gamma\right\}$. Используя формулу (1), имеем

$$
\begin{aligned}
F_{x\left(t_{2}\right)-x\left(t_{1}\right)=} & \frac{1}{2 \pi} \frac{1}{\sqrt{\operatorname{det} K}} \int_{B} d u_{1} d u_{2} \exp \left[-\frac{1}{2}\left(k_{11}^{(-1)} u_{1}^{2}+k_{12}^{(-1)} u_{1} u_{2}+\right.\right. \\
& \left.\left.+k_{21}^{(-1)} u_{2} u_{1}+k_{22}^{(-1)} u_{2}^{2}\right)\right]
\end{aligned}
$$

Элементы обратной матрицы $K^{-1}$ в этом случае просто вычисляются и имеют вид

$$
k_{11}^{(-1)}=k_{22}^{(-1)}=\frac{k_{11}}{\operatorname{det} K}, \quad k_{12}^{(-1)}=k_{21}^{(-1)}=-\frac{k_{12}}{\operatorname{det} K},
$$

где

$$
\begin{gathered}
k_{11}=\frac{1}{2 m \omega \operatorname{sh} \frac{\beta \omega}{2}} \operatorname{ch} \frac{\beta \omega}{2}, \quad k_{12}=\frac{1}{2 m \omega \operatorname{sh} \frac{\beta \omega}{2}} \operatorname{ch}\left(\omega\left|t_{1}-t_{2}\right|-\frac{\beta \omega}{2}\right), \\
\operatorname{det} K=\frac{1}{4 m^{2} \omega^{2} \operatorname{sh}^{2} \frac{\beta \omega}{2}}\left[\operatorname{ch}^{2} \frac{\beta \omega}{2}-\operatorname{ch}^{2}\left(\omega\left|t_{1}-t_{2}\right|-\frac{\beta \omega}{2}\right)\right] .
\end{gathered}
$$

После подстановки последних выражений в формулу (3) и некоторых элементарных преобразований получаем

$$
\begin{aligned}
F_{x\left(t_{2}\right)-x\left(t_{1}\right)}= & \sqrt{\frac{m \omega \operatorname{sh} \frac{\beta \omega}{2}}{2 \pi\left[\operatorname{ch} \frac{\beta \omega}{2}-\operatorname{ch}\left(\frac{\beta \omega}{2}-\omega\left(t_{2}-t_{1}\right)\right)\right]}} \times \\
& \times \int_{-\infty}^{\gamma} d u \exp \left[-\frac{u^{2}}{2} \frac{m \omega \operatorname{sh} \frac{\beta \omega}{2}}{\operatorname{ch} \frac{\beta \omega}{2}-\operatorname{ch}\left(\frac{\beta \omega}{2}-\omega\left(t_{2}-t_{1}\right)\right)}\right] .
\end{aligned}
$$

Следовательно, случайная величина $G(x)=x\left(t_{2}\right)-x\left(t_{1}\right)$ нормально распределена с нулевым средним и дисперсией

$$
\frac{\operatorname{ch} \frac{\beta \omega}{2}-\operatorname{ch}\left(\frac{\beta \omega}{2}-\omega\left(t_{2}-t_{1}\right)\right)}{m \omega \operatorname{sh} \frac{\beta \omega}{2}}
$$




\section{2. НЕДИФФЕРЕНЦИРУЕМОСТЬ БОГОЛЮБОВСКИХ ТРАЕКТОРИЙ}

Рассмотрим свойства носителя меры Боголюбова в пространстве $C^{\circ}[0, \beta]$. Оказыва-

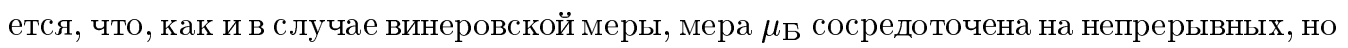
не на непрерывно дифференцируемых путях. Таким образом, мера Боголюбова, наряду с мерой Винера, дает еше один важный пример везде непрерывных, но почти всюду недифференцируемых функций.

Введем множество

$$
C_{h}^{\gamma}\left(t, t^{\prime}\right)=\left\{x \in X:\left|x(t)-x\left(t^{\prime}\right)\right| \leqslant h\left|t-t^{\prime}\right|^{\gamma}\right\}
$$

где $h>0,0<\gamma \leqslant 1, t, t^{\prime} \in[0, \beta]$. Попробуем найти боголюбовскую меру этого множества. Воспользуемся формулой (1). Тогда можно записать, что

$$
\mu_{\mathrm{Б}}\left\{C_{h}^{\gamma}\left(t, t^{\prime}\right)\right\}=\frac{1}{2 \pi \sqrt{\operatorname{det} K}} \int_{B} d u_{1} d u_{2} e^{-\frac{1}{2}\left(u, K^{-1} u\right)},
$$

где $B=\left\{\left(u_{1}, u_{2}\right) \in R^{2}:\left|u_{1}-u_{2}\right| \leqslant h\left|t-t^{\prime}\right|^{\gamma}\right\}$. Матрица $K$, входяшая в формулу (5), совпадает с матрицей $K$, использованной в предыдущем разделе в формуле (3). Выполнив в интеграле (5) подходящую линейную замену переменных интегрирования, получим

$$
\mu_{\mathrm{5}}\left\{C_{h}^{\gamma}\left(t, t^{\prime}\right)\right\}=\frac{1}{\sqrt{2 \pi}} \int_{-a}^{a} e^{-\frac{v^{2}}{2}} d v,
$$

где

$$
a=\sqrt{\frac{m \omega \operatorname{sh} \frac{\beta \omega}{2}}{\operatorname{ch} \frac{\beta \omega}{2}-\operatorname{ch}\left(\frac{\beta \omega}{2}-\omega\left|t-t^{\prime}\right|\right)}} h\left|t-t^{\prime}\right|^{\gamma} .
$$

Из формулы (6) следует оценка сверху для искомой меры,

$$
\mu_{\mathrm{Б}}\left\{C_{h}^{\gamma}\left(t, t^{\prime}\right)\right\} \leqslant \sqrt{\frac{2}{\pi}} a .
$$

Введем теперь множества

$$
\mathrm{C}_{h}^{\gamma}(t)=\bigcap_{t^{\prime} \in[0, \beta]} C_{h}^{\gamma}\left(t, t^{\prime}\right)=\left\{x \in X:\left|x(t)-x\left(t^{\prime}\right)\right| \leqslant h\left|t-t^{\prime}\right|^{\gamma} \text { для всех } t^{\prime} \in[0, \beta]\right\}
$$

и

$$
\mathrm{C}_{h}^{\gamma}=\bigcap_{t \in[0, \beta]} C_{h}^{\gamma}(t)=\left\{x \in X:\left|x(t)-x\left(t^{\prime}\right)\right| \leqslant h\left|t-t^{\prime}\right|^{\gamma} \text { для всех } t, t^{\prime} \in[0, \beta]\right\} .
$$

Можно доказать [3], что множества $C_{h}^{\gamma}\left(t, t^{\prime}\right), C_{h}^{\gamma}(t)$ и $C_{h}^{\gamma}$ являются замкнутыми подмножествами в $X=C^{\circ}[0, \beta]$.

5 Теоретическая и математическая физика, т. 127, № 1, 2001 г. 
Рассмотрим последовательность $\left\{t_{k}\right\}$ точек из сегмента $[0, \beta]$ таких, что они не совпадают с точкой $t$ и $t_{k} \rightarrow t$ при $k \rightarrow \infty$. Тогда из определения (8), оценки (7) и свойства выпуклости вниз функции сh $x$ следует, что

$$
\mu_{\mathrm{Б}}\left\{C_{h}^{\gamma}(t)\right\} \leqslant \mu_{\mathrm{Б}}\left\{C_{h}^{\gamma}\left(t, t_{k}\right)\right\} \leqslant \sqrt{\frac{2 m \operatorname{sh} \frac{\beta \omega}{2}}{\pi \operatorname{sh}\left(\frac{\beta \omega}{2}-\omega\left|t-t_{k}\right|\right)}} h\left|t-t_{k}\right|^{\gamma-\frac{1}{2}} .
$$

Из последнего неравенства при $\gamma>1 / 2$ получается, что $\mu_{\mathrm{Б}}\left\{C_{h}^{\gamma}(t)\right\}=0$, а следовательно, и

$$
\mu_{\mathrm{5}}\left\{C_{h}^{\gamma}\right\}=0 .
$$

Напомним, что функция $x:[0, \beta] \mapsto R$ называется непрерывной по Гельдеру порядка $\gamma$ тогда и только тогда, когда сушествует положительная постоянная $h$ такая, что $\mid x(t)-$ $x\left(t^{\prime}\right)|\leqslant h| t-\left.t^{\prime}\right|^{\gamma}$ для всех $t, t^{\prime} \in[0, \beta]$ и $\gamma \in(0, t]$. Поскольку

$$
\Gamma^{\gamma} \equiv\{x \in X: x-\text { непрерывная по Гельдеру функция порядка } \gamma\}=\bigcup_{h=1}^{\infty} C_{h}^{\gamma},
$$

то в силу условия (9) следует, что множество $\Gamma^{\gamma}$ при $1 / 2<\gamma \leqslant 1$ является борелевским подмножеством $X$ с боголюбовской мерой (или вероятностью) ноль. Иными словами, почти всюду по мере боголюбовские траектории не являются непрерывными по Гельдеру порядка $\gamma>1 / 2$ (а следовательно, не могут быть непрерывно дифференцируемы).

Пусть $0 \leqslant t \leqslant \beta$ и пусть $D_{t}=\left\{x \in X: x^{\prime}(t)\right.$ существует $\}$, где $x^{\prime}(t)$ обозначает обычную производную функции $x$ по $t$ при $t \in(0, \beta)$ и соответственно одностороннюю производную при $t=0$ или $t=\beta$. Тогда можно показать [3], что $D_{t} \subset \bigcup_{h=1}^{\infty} C_{h}^{1}(t)$, откуда следует, что $\mu_{\text {Б }}\left(D_{t}\right)=0$.

Определим функцию $F=X \times[0, \beta] \mapsto R$ соотношением

$$
F(x, t)= \begin{cases}1, & \text { если } x^{\prime}(t) \text { сушествует (как конечная величина) } \\ 0 & \text { в противном случае. }\end{cases}
$$

Можно показать [3], что функция $F$ измерима как функция от $x$ и $t$. Тогда по теореме Фубини

$$
\int_{X}\left(\int_{0}^{\beta} F(x, t) d t\right) d \mu_{\mathrm{Б}}(x)=\int_{0}^{\beta}\left(\int_{X} F(x, t) d \mu_{\mathrm{Б}}(x)\right) d t=\int_{0}^{\beta} \mu_{\mathrm{Б}}\left(D_{t}\right) d t=0 .
$$

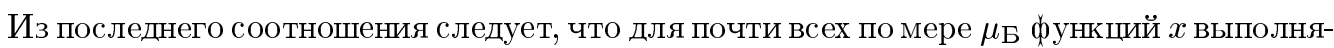
ется равенство

$$
\int_{0}^{\beta} F(x, t) d t=0
$$

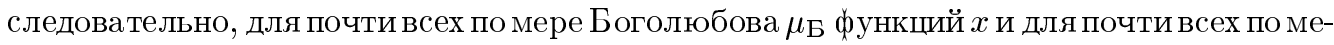
ре Лебега значений $t$ выполняется равенство $F(x, t)=0$. Таким образом, доказано, что 
траектории $x \in X$ с вероятностью 1 дифференцируемы самое большее на подмножестве $[0, \beta]$ с лебеговой мерой ноль.

Поскольку любая функция $x$ ограниченной вариации на любом интервале почти всюду по мере Лебега дифференцируема на этом интервале [4], то боголюбовские траектории с вероятностью 1 на любом подынтервале отрезка $[0, \beta]$ имеют неограниченную вариацию.

Описанные в этом разделе свойства боголюбовских траекторий полностью совпадают с соответствуюшими свойствами винеровских траекторий. Однако имеются и различия, которые проявятся далее.

\section{3. МАСШТАБНЫЕ ПРЕОБРАЗОВАНИЯ В ПРОСТРАНСТВЕ БОГОЛЮБОВА}

Важное значение в теории фейнмановских континуальных интегралов имеет масштабное преобразование в соответствуюшем функшиональном пространстве $x \mapsto \sigma x$, где параметр $\sigma \in C$. При этом сушественную роль играет известная теорема Леви о квадратичной вариации винеровских траекторий [5] и специальный случай, исследованный несколько позднее в работе [6]. Имея в виду возможность аналитического продолжения по температуре или массе в боголюбовском континуальном интеграле, интересно применить схему Леви для случая меры Боголюбова.

Введем квадратичные вариации Леви траекторий. Рассмотрим разбиение П сегмента $[0, \beta], 0=t_{0}<t_{1}<\cdots<t_{k}=\beta$ и функцию $x \in X$. Определим функцию

$$
S_{\Pi}(x)=\sum_{j=1}^{k}\left[x\left(t_{j}\right)-x\left(t_{j-1}\right)\right]^{2} .
$$

В случае разбиения сегмента $[0, \beta]$ на $k$ равных интервалов будем просто писать $S_{k}(x)$ вместо $S_{\Pi}(x)$. Заметим, что для достаточно гладких траекторий, удовлетворяюших, например, условию Липшица с постоянной $k$,

$$
\lim _{n \rightarrow \infty} S_{2^{n}}(x)=0 .
$$

Из предыдушего раздела, однако, известно, что мера Боголюбова сосредоточена на недифференцируемых траекториях. Поэтому, как и для винеровской меры, следует ожидать, что для боголюбовских траекторий условие (11) не будет вьполняться.

Tеорема 1. Боголюбовские траектории $x \in X$ почти всюду удовлетворяют соотношению

$$
\lim _{n \rightarrow \infty} S_{2^{n}}(x)=\frac{\beta}{m} .
$$


ДокАЗАТЕЛЬСтво. Покажем сначала, что для любого достаточно большого натурального числа $N$

$$
I_{N} \equiv\left\|S_{N}-\frac{\beta}{m}\right\|_{2}^{2}=\frac{2 \beta^{2}}{m^{2}} \frac{1}{N}+O\left(\frac{1}{N^{2}}\right)
$$

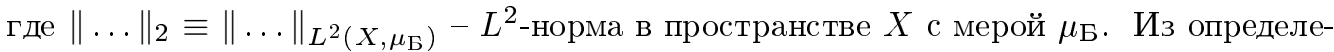
ния (10) следует, что

$$
S_{N}(x)=\sum_{j=1}^{N}\left[x\left(t_{j}\right)-x\left(t_{j-1}\right)\right]^{2}, \quad t_{j}=\frac{j \beta}{N}, \quad j=0,1, \ldots, N .
$$

Для искомой величины $I_{N}$ имеем

$$
\begin{aligned}
I_{N} & =\int_{X}\left(S_{N}(x)-\frac{\beta}{m}\right)^{2} d \mu_{\mathrm{Б}}(x)= \\
& =\int_{X} S_{N}^{2}(x) d \mu_{\mathrm{Б}}(x)-\frac{2 \beta}{m} \int_{X} S_{N}(x) d \mu_{\mathrm{Б}}(x)+\frac{\beta^{2}}{m^{2}} .
\end{aligned}
$$

Из первого раздела известно, что случайная величина $x\left(t_{j}\right)-x\left(t_{j-1}\right)$ распределена с нулевым средним и дисперсией (4), поэтому

$$
\begin{aligned}
\int_{X} S_{N}(x) d \mu_{\text {Б }}(x) & =\sum_{j=1}^{N} \frac{\operatorname{ch} \frac{\beta \omega}{2}-\operatorname{ch}\left(\frac{\beta \omega}{2}-\omega\left|t_{j}-t_{j-1}\right|\right)}{m \omega \operatorname{sh} \frac{\beta \omega}{2}}= \\
& =N \frac{\operatorname{ch} \frac{\beta \omega}{2}-\operatorname{ch}\left(\frac{\beta \omega}{2}-\frac{\beta \omega}{N}\right)}{m \omega \operatorname{sh} \frac{\beta \omega}{2}} .
\end{aligned}
$$

В частности, при $N \rightarrow \infty$ имеем

$$
\int_{X} S_{N}(x) d \mu_{\mathrm{Б}}(x)=\frac{\beta}{m}-\frac{\beta^{2} \omega}{2 m} \operatorname{cth} \frac{\beta \omega}{2} \frac{1}{N}+O\left(\frac{1}{N^{2}}\right) .
$$

Вычислим теперь интеграл от $S_{N}^{2}(x)$, входяший в формулу (13):

$$
\begin{aligned}
\int_{X} S_{N}^{2}(x) d \mu_{\mathrm{Б}}(x)= & \sum_{m, n=1}^{N} \int_{X} d \mu_{\mathrm{Б}}(x)\left[x^{2}\left(t_{n}\right) x^{2}\left(t_{m}\right)+2 x^{2}\left(t_{n}\right) x^{2}\left(t_{m-1}\right)+\right. \\
& +x^{2}\left(t_{n-1}\right) x^{2}\left(t_{m-1}\right)-4 x^{2}\left(t_{n}\right) x\left(t_{m}\right) x\left(t_{m-1}\right)- \\
& \left.-4 x^{2}\left(t_{n-1}\right) x\left(t_{m}\right) x\left(t_{m-1}\right)+4 x\left(t_{n}\right) x\left(t_{n-1}\right) x\left(t_{m}\right) x\left(t_{m-1}\right)\right] .
\end{aligned}
$$

При вычислении интегралов от отдельных слагаемых в правой части (14) необходимо воспользоваться теоремой Вика и соответствующими табличными значениями конечных сумм [7]. Например,

$$
\int_{X} d \mu_{\mathrm{5}}(x) x^{2}\left(t_{n}\right) x^{2}\left(t_{m}\right)=B^{2}\left(t_{n}, t_{n}\right)+2 B^{2}\left(t_{n}, t_{m}\right) .
$$


Используя формулу (2), найдем

$$
\begin{aligned}
\sum_{n, m=1}^{N} \int_{X} d \mu_{\text {Б }}(x) x^{2}\left(t_{n}\right) x^{2}\left(t_{m}\right)= & \left(2 m \omega \operatorname{sh} \frac{\beta \omega}{2}\right)^{-2} \times \\
& \times\left(N^{2} \operatorname{ch}^{2} \frac{\beta \omega}{2}+N^{2}+N \operatorname{sh}(\beta \omega) \operatorname{cth} \frac{\beta \omega}{N}\right) .
\end{aligned}
$$

Вычисляя соответствуюшим образом остальные слагаемые в правой части (14), получим

$$
\begin{aligned}
\int_{X} S_{N}^{2}(x) d \mu_{\text {Б }}(x)= & \left(2 m \omega \operatorname{sh} \frac{\beta \omega}{2}\right)^{-2}\left[4 N^{2} \operatorname{ch}^{2} \frac{\beta \omega}{2}+4 N^{2} \operatorname{ch}^{2}\left(\frac{\beta \omega}{2}-\frac{\beta \omega}{N}\right)-\right. \\
& -8 N^{2} \operatorname{ch} \frac{\beta \omega}{2} \operatorname{ch}\left(\frac{\beta \omega}{2}-\frac{\beta \omega}{N}\right)+6 N^{2}-8 N^{2} \operatorname{ch} \frac{\beta \omega}{N}+ \\
& +2 N(N-1) \operatorname{ch} \frac{2 \beta \omega}{N}+2 N+2 N \operatorname{ch}\left(\beta \omega-\frac{2 \beta \omega}{N}\right)+ \\
& \left.+6 N \operatorname{sh}(\beta \omega) \frac{\operatorname{ch} \frac{\beta \omega}{N}}{\operatorname{sh} \frac{\beta \omega}{N}}-8 N \frac{\operatorname{sh}(\beta \omega)}{\operatorname{sh} \frac{\beta \omega}{N}}+2 N \frac{\operatorname{sh}\left(\beta \omega-\frac{\beta \omega}{N}\right)}{\operatorname{sh} \frac{\beta \omega}{N}}\right] .
\end{aligned}
$$

Имея в виду предельный переход $N \rightarrow \infty$, из формулы (15) находим

$$
\int_{X} S_{N}^{2}(x) d \mu_{\mathrm{Б}}(x)=\frac{\beta^{2}}{m^{2}}+\frac{\beta^{2}}{2 m^{2}} \frac{2 \operatorname{ch}(\beta \omega)-\beta \omega \operatorname{sh}(\beta \omega)-2}{\operatorname{sh}^{2} \frac{\beta \omega}{2}} \frac{1}{N}+\varepsilon_{N}
$$

где положительное число $\varepsilon_{N} \sim O\left(1 / N^{2}\right)$. В итоге получаем для искомой величины $I_{N}$ требуемое соотношение (12). Из равенства (12), в частности, следует, что

$$
\left\|S_{2^{n}}-\frac{\beta}{m}\right\|_{2}^{2}=\frac{\beta^{2}}{m^{2}} \frac{1}{2^{n-1}}+\varepsilon_{2^{n}} .
$$

Рассмотрим множество

$$
E_{n}=\left\{x \in X:\left|S_{2^{n}}(x)-\frac{\beta}{m}\right| \geqslant \frac{\beta}{m} \frac{1}{2^{\frac{n}{3}}}+\mu_{n}\right\}
$$

где

$$
\mu_{n}=\frac{\beta}{m} \frac{1}{2^{\frac{n}{3}}}\left(\sqrt{1+\frac{m^{2}}{\beta^{2}} 2^{n-1} \varepsilon_{n}}-1\right) \sim O\left(\frac{1}{2^{\frac{4 n}{3}+1}}\right) .
$$

Докажем, что

$$
\mu_{\text {Б }}\left(E_{n}\right) \leqslant \frac{2}{2^{\frac{n}{3}}} .
$$


Допустим противное, тогда

$$
\begin{aligned}
\int_{X}\left|S_{2^{n}}(x)-\frac{\beta}{m}\right|^{2} d \mu_{\text {Б }}(x) & \geqslant \int_{E_{n}}\left|S_{2^{n}}(x)-\frac{\beta}{m}\right|^{2} d \mu_{\text {Б }}(x)> \\
& >\left(\frac{\beta}{m} \frac{1}{2^{\frac{n}{3}}}+\mu_{n}\right)^{2} \frac{2}{2^{\frac{n}{3}}}=\frac{\beta^{2}}{m^{2}} \frac{1}{2^{n-1}}+\varepsilon_{2^{n}},
\end{aligned}
$$

что противоречит (16). Положим

$$
F_{n}=\bigcup_{k=n}^{\infty} E_{k} .
$$

Тогда из (18) следует, что

$$
\mu_{\mathrm{Б}}\left(F_{n}\right) \leqslant \sum_{k=n}^{\infty} \mu_{\mathrm{Б}}\left(E_{k}\right) \leqslant \frac{c}{2^{\frac{n}{3}}}
$$

где $c=2^{4 / 3}\left(2^{1 / 3}-1\right)^{-1}$. Теперь из (17) вытекает, что для $x \in X \backslash F_{n}=\bigcap_{k=n}^{\infty} E_{k}^{\text {c }}$, где $E_{k}^{\mathrm{c}}$ обозначает дополнение множества $E_{k}$ в $X$, выполняется неравенство

$$
\left|S_{2^{k}}(x)-\frac{\beta}{m}\right|<\frac{\beta}{m} 2^{-\frac{k}{3}}
$$

при любых $k=n, n+1, \ldots$ Следовательно, если найдется такое число $n$, что $x \notin F_{n}$, то

$$
\lim _{k \rightarrow \infty} S_{2^{k}}(x)=\frac{\beta}{m} .
$$

Таким образом, условие (20) выполняется при всех $x$, за исключением, возможно, множества $F=\bigcap_{n=1}^{\infty} F_{n}$. Но из неравенства (19) следует, что для любого $n$

$$
\mu_{\mathrm{Б}}(F) \leqslant \mu_{\mathrm{Б}}\left(F_{n}\right) \leqslant \frac{c}{2^{\frac{n}{3}}},
$$

т.е. $\mu_{\mathrm{Б}}(F)=0$. Теорема 1 доказана.

Для заданного положительного числа $\sigma$ рассмотрим множество

$$
\Omega_{\sigma}=\left\{x \in X: \lim _{n \rightarrow \infty} S_{2^{n}}(x)=\sigma^{2} \frac{\beta}{m}\right\}
$$

Обозначим через $\mu_{\mathrm{Б}}^{\sigma}(\sigma>0)$ образ меры $\mu_{\mathrm{Б}} \equiv \mu_{\mathrm{Б}}^{1}$ при отображении $\varphi_{\sigma}: X \mapsto X, \varphi_{\sigma}=$ $\sigma x$. Мера $\mu_{\mathrm{Б}}^{\sigma}=\mu_{\mathrm{Б}}^{1} \circ \sigma^{-1}$ определена на борелевской $\sigma$-алгебре $\mathcal{B}(X)$ и для любого $B \in \mathcal{B}$ выполнено соотношение $\mu_{\mathrm{5}}^{\sigma}(B)=\mu_{\mathrm{5}}^{1}\left(\sigma^{-1} B\right)$. 
УТВЕРЖДЕНИЕ 1 [3].

А. Множество $\Omega_{\sigma}$ измеримо по Борелю для любого $\sigma>0$.

Б. Для любих $\sigma_{1}, \sigma_{2}>0$ имеет место соотношение $\sigma_{2} \Omega_{\sigma_{1}}=\Omega_{\sigma_{1} \sigma_{2}}$ и, в частности, для любого $\sigma>0 \quad \sigma \Omega_{1}=\Omega_{\sigma}$.

B. $\mu_{\mathrm{Б}}^{\sigma}\left(\Omega_{\sigma}\right)=1 \partial \Omega_{\text {я }}$ любого $\sigma>0$.

Г. Если $\sigma_{1} \neq \sigma_{2} \quad\left(\sigma_{1}, \sigma_{2}>0\right)$, то $\Omega_{\sigma_{1}} \cap \Omega_{\sigma_{2}}=\varnothing$, т.е. меры $\mu_{Б}^{\sigma_{1}}$ и $\mu_{Б}^{\sigma_{2}}$ взаимно ортогональны.

Из п. В сформулированного утверждения следует, что множество $\Omega_{\sigma}$ является множеством полной $\mu_{\mathrm{Б}}^{\sigma}$-меры. Вслед за авторами книги [3] будем говорить, что мера $\mu_{\mathrm{Б}}^{\sigma}$ сконщентрирована на множестве $\Omega_{\sigma}$. Заметим, что $\operatorname{supp} \mu_{\mathrm{5}}^{\sigma}=X$ для любого $\sigma>0$ и $\Omega_{\sigma} \subset X$.

Назовем подмножество $A$ из $X$ масштабно-инвариантным измеримым множеством, если $\sigma A \in \mathcal{S}_{1}$ для всех $\sigma>0$, где $\mathcal{S}_{1}-\sigma$-алгебра измеримых по мере Боголюбова $\mu_{\text {Б мно- }}^{1}$ жеств пространства $X$. Масштабно-инвариантное измеримое множество $N$ называется нуль-масштабно-инвариантным множеством, если $\mu_{5}^{1}(\sigma N)=0$ для всех $\sigma>0$. Классы масштабно-инвариантных и нуль-масштабно-инвариантных множеств будем обозначать как $\mathcal{S}$ и $\mathcal{N}$, соответственно. Обозначим через $\mathcal{S}_{\sigma} \sigma$-алгебру, полученную пополнением измеримого пространства $\left(X, \mathcal{B}(X), \mu_{\mathrm{Б}}^{\sigma}\right)$, а через $\mathcal{N}_{\sigma}$ - класс $\mu_{\mathrm{Б}}^{\sigma}$-нуль множеств. Можно показать, что $\mathcal{N}_{\sigma}=\sigma \mathcal{N}_{1}, \mathcal{S}_{\sigma}=\sigma \mathcal{S}_{1}$ и $\mu_{\mathrm{b}}^{\sigma}(E)=\mu_{\text {Б }}^{1}\left(\sigma^{-1} E\right)$ для любого $E \in \mathcal{S}_{\sigma}$. Кроме того, алгебра $\mathcal{S}$ есть $\sigma$-алгебра и $\mathcal{S}=\bigcap_{\sigma>0} \mathcal{S}_{\sigma}$, а также $\mathcal{N}=\bigcap_{\sigma>0} \mathcal{N}_{\sigma}$. Легко видеть, что для каждого $\sigma>0 \quad \mathcal{B}(X) \subset \mathcal{S} \subset \mathcal{S}_{\sigma}$. Множество $E \in \mathcal{S}$ тогда и только тогда, когда $E \cap \Omega_{\sigma} \in \mathcal{S}_{\sigma}$ для любого $\sigma>0$. Множество $N \in \mathcal{N}$ тогда и только тогда, когда $N \cap \Omega_{\sigma} \in \mathcal{N}_{\sigma}$ для любого $\sigma>0$.

Структура масштабно-инвариантных и нуль-масштабно-инвариантных множеств определяется следуюшим утверждением.

УТВЕРЖДЕНИЕ 2 [3].

А. Множество $E \in \mathcal{S}$ тогда и только тогда, когда оно может быть представлено в виде

$$
E=\left(\bigcup_{\sigma>0} E_{\sigma}\right) \cup L
$$

где каждое множество $E_{\sigma}$ является $\mu_{Б}^{\sigma}$-измеримым подмножеством множества $\Omega_{\sigma}$, а $L$ - произвольное подмножество множества

$$
X \backslash \bigcup_{\sigma>0} \Omega_{\sigma} .
$$

Для любого множества $E$ вида (21) имеем $\mu_{\mathrm{Б}}^{\sigma}(E)=\mu_{\mathrm{Б}}^{\sigma}\left(E_{\sigma}\right)$ для всех $\sigma>0$.

Б. Множество $N \in \mathcal{N}$ тогда и только тогда, когда оно может бить представлено в виде

$$
N=\left(\bigcup_{\sigma>0} N_{\sigma}\right) \cup L
$$

где каждое множество $N_{\sigma}$ является $\mu_{\mathrm{Б}}^{\sigma}$-измеримим подмножеством множества $\Omega_{\sigma}$, a $L$ - произвольное подмножество множества (22). 


\section{4. ПОЛУГРУППЫ ПО МЕРЕ БОГОЛЮБОВА}

Обозначим через $\mathcal{L}(X)$ пространство ограниченных линейных операторов, действующих в банаховом пространстве $X$. Напомним, что семейство операторов $\{T(t): 0 \leqslant$ $t<\infty\}$ в $\mathcal{L}(X)$ называется сильно непрерывной полугруппой операторов на $X$, если $T(0)=I, T(t, s)=T(t) T(s)$ для всех $t, s \in[0, \infty)$ и для каждого $x \in X$ отображение $t \mapsto T(t) x$ из $[0, \infty)$ в $X$ непрерьвно. Известно [8], что в случае сильно непрерывной полугруппы производящий оператор (генератор) этой полугруппы

$$
L=\lim _{\epsilon \rightarrow 0} \frac{1}{\epsilon}(T(\epsilon)-I)
$$

имеет область определения $D(L)$, плотную в $X$, и является замкнутым линейным оператором, причем для $f \in D(L)$

$$
\lim _{\epsilon \rightarrow 0} \frac{1}{\epsilon}(T(t+\epsilon)-T(t)) f=L T(t) f=T(t) L f
$$

Можно показать [9], что если задана сильно непрерывная полугруппа $\{T(t): 0 \leqslant t<$ $\infty\}$ с генератором $L$ и задан произвольный вектор $f \in D(L)$, то функция $u(t)=T(t) f \in$ $X$ непрерывно дифференцируема на полуинтервале $[0, \infty)$, удовлетворяет начальному условию $u(0)=f$ и для всех $t>0$ выполняется дифференциальное уравнение $d u / d t=$ $L u$.

В случае гауссовых мер сушествует универсальный пример сильно непрерывной полугруппы - полугруппа Орнштейна-Уленбека. Пусть $\mu$ - центрированная гауссова мера на локально-выпуклом пространстве $X$. Полугруппа Орнштейна-Уленбека на пространстве $L^{p}(\mu)$ определяется формулой

$$
T(t) f(x)=\int_{X} f\left(e^{-t} x+\sqrt{1-e^{2 t}} y\right) d \mu(y)
$$

Доказывается [10], что для всякого $p \geqslant 1$ семейство операторов $\{T(t): 0 \leqslant t<\infty\}$, заданных формулой (23), образует сильно непрерывную полугруппу на банаховом пространстве $L^{p}(\mu)$ с операторной нормой

$$
\|T(t)\|_{\mathcal{L}\left(L^{p}(\mu)\right)}=1
$$

Кроме того, при $p=2$ операторы $T(t)$ неотрицательны.

В случае меры Боголюбова можно получить вид генератора полугруппы Орнштейна-Уленбека, считая, что функция $f \in C_{0}^{\infty}(R)$, где $C_{0}^{\infty}(R)$ - пространство бесконечно дифференцируемых функций с компактным носителем в $R$,

$$
T(t) f(x)=\frac{1}{\sqrt{\frac{\pi}{m \omega} \operatorname{cth} \frac{\beta \omega}{2}}} \int_{-\infty}^{\infty} f\left(e^{-t} x+\sqrt{1-e^{-2 t}} y\right) \exp \left(-\frac{m \omega y^{2}}{\operatorname{cth} \frac{\beta \omega}{2}}\right) d y
$$


С делав замену переменной интегрирования $e^{-t} x+\sqrt{1-e^{-2 t}} y=z$, получим

$$
\begin{aligned}
\frac{T(t) f(x)-f(x)}{t}=\frac{1}{t} & \left\{\sqrt{\frac{m \omega \operatorname{th} \frac{\beta \omega}{2}}{\pi\left(1-e^{-2 t}\right)}} \int_{-\infty}^{\infty}(f(z)-f(x)) \times\right. \\
& \left.\times \exp \left[-\frac{m \omega \operatorname{th} \frac{\beta \omega}{2}}{1-e^{-2 t}}\left(z-e^{-t} x\right)^{2}\right] d z\right\}
\end{aligned}
$$

Разложим теперь функцию $f(z)$ под знаком интеграла в последнем выражении, используя теорему Тейлора,

$$
f(z)=f(x)+f^{\prime}(x)(z-x)+\frac{1}{2} f^{\prime \prime}(x)(z-x)^{2}+\frac{1}{6} f^{(3)}(x)(z-x)^{3}+\frac{f^{(4)}(\xi)}{24}(z-x)^{4} .
$$

Тогда, вычисляя элементарные гауссовы интегралы, находим

$$
\frac{T(t) f(x)-f(x)}{t}=-x f^{\prime}(x)+\frac{1}{2} f^{\prime \prime}(x) \frac{1}{2 t} \frac{1-e^{-2 t}}{m \omega \text { th } \frac{\beta \omega}{2}}+o(t),
$$

т.е. генератор полугруппы (23) в случае меры Боголюбова задается соотношением

$$
L=-x \frac{d}{d x}+\frac{\operatorname{cth} \frac{\beta \omega}{2}}{2 m \omega} \frac{d^{2}}{d x^{2}} .
$$

Рассмотрим теперь семейство операторов $\{T(\beta): 0 \leqslant \beta<\infty\}$, действующих в пространстве $L^{2}(R)$ по формуле

$$
(T(\beta) f)(x)=\int_{X} d \mu_{\mathrm{5}}(y) f\left(\int_{0}^{\beta} y(t) d t+x\right) .
$$

Очевидно, что $T(0)=I$. Кроме того, используя формулы интегрирования по гауссовым мерам [11], имеем

$$
(T(\beta) f)(x)=\sqrt{\frac{m \omega^{2}}{2 \pi \beta}} \int_{-\infty}^{\infty} f(y) \exp \left[-\frac{(y-x)^{2} m \omega^{2}}{2 \beta}\right] d y .
$$

Формула (25) является известной формулой свободной полугруппы для уравнения теплопроводности. Таким образом, семейство операторов (24) действительно является сильно непрерывной полугруппой в пространстве $L^{2}(R)$. При этом генератор этой полугруппы имеет вид

$$
L=\frac{1}{2 m \omega^{2}} \frac{d^{2}}{d x^{2}}
$$

и функция $u(\beta, x)=(T(\beta) f)(x)$ для любой функции $f \in L^{2}(R)$ определяет решение уравнения Блоха

$$
\frac{\partial u}{\partial \beta}=\frac{1}{2 m \omega^{2}} \frac{\partial^{2} u}{\partial x^{2}}
$$

с начальным условием $u(0, x)=f(x)$. Из формулы $(24)$ вытекает следующее соотношение, выражающее связь между мерами Боголюбова и Винера:

$$
\int_{C^{\circ}\left[0, m \omega^{2} t\right]} f\left(x+\int_{0}^{m \omega^{2} t} y(\tau) d \tau\right) d \mu_{\text {Б }}(y)=\int_{C_{0}^{t}} f(y(t)+x) d \mu_{\mathrm{W}}(y),
$$

где $C_{0}^{t}$ - пространство непрерывных на $[0, t]$ функций, равных нулю в нуле. 


\section{5. НЕЗАВИСИМЫЕ ПРИРАШЕНИЯ}

Классический винеровский процесс на отрезке $[a, b]$ имеет независимые приращения, т.е. для любых $a<t_{1}<t_{2}<\cdots<t_{n} \leqslant b$ соответствуюшие случайные величины $\xi_{t_{2}}-$ $\xi_{t_{1}}, \ldots, \xi_{t_{n}}-\xi_{t_{n-1}}$ являются независимыми. В силу гауссовости винеровского процесса для доказательства этого утверждения достаточно проверить лишь попарную независимость таких приращений. В случае меры Боголюбова приращения $x\left(t_{i}\right)-x\left(t_{i-1}\right)$, $i=1,2, \ldots, n$, не являются независимыми, что сушественно затрудняет анализ соответствуюшего случайного процесса. Однако установленная в разделе 4 связь между мерами Винера и Боголюбова позволяет построить систему независимых приращений и в случае боголюбовского случайного процесса.

Рассмотрим случайную величину

$$
y(t)=\lambda x(t)+\int_{0}^{t} x(\tau) d \tau, \quad 0 \leqslant t \leqslant \beta,
$$

где постоянная $\lambda$ будет определена ниже. Для математического ожидания $M(y(t) y(s))$ имеем

$$
\begin{aligned}
M(y(t) y(s))= & {\left[\frac{\lambda^{2}}{2 m \omega \operatorname{sh} \frac{\beta \omega}{2}}-\frac{1}{2 m \omega^{3} \operatorname{sh} \frac{\beta \omega}{2}}\right] \operatorname{ch}\left(\omega|t-s|-\frac{\beta \omega}{2}\right)+} \\
& +\frac{1}{2 m \omega^{2} \operatorname{sh} \frac{\beta \omega}{2}}\left[2 \operatorname{sh} \frac{\beta \omega}{2} \min (s, t)-\frac{1}{\omega} \operatorname{ch} \frac{\beta \omega}{2}+\right. \\
& \left.+\frac{1}{\omega} \operatorname{ch}\left(\omega s-\frac{\beta \omega}{2}\right)+\frac{1}{\omega} \operatorname{ch}\left(\omega t-\frac{\beta \omega}{2}\right)\right]+ \\
& +\frac{\lambda}{2 m \omega^{2} \operatorname{sh} \frac{\beta \omega}{2}}\left[2 \operatorname{sh} \frac{\beta \omega}{2}+\operatorname{sh}\left(\omega s-\frac{\beta \omega}{2}\right)+\operatorname{sh}\left(\omega t-\frac{\beta \omega}{2}\right)\right] .
\end{aligned}
$$

Выбрав теперь $\lambda=\omega^{-1}$, легко установить, что

$$
M[(y(t)-y(s))(y(\tau)-y(\sigma))]=0 \text { при } s<t<\sigma<\tau .
$$

Таким образом, гауссовость случайного процесса Боголюбова позволяет сформулировать полученный результат в виде следуюшей теоремы.

Теорема 2. Гауссов случайный прочесс с мерой Боголюбова имеет независимые приращения, т.е. для любых $0<t_{1}<t_{2}<\cdots<t_{n} \leqslant \beta$ случайные величины $y\left(t_{2}\right)-y\left(t_{1}\right), \ldots, y\left(t_{n}\right)-y\left(t_{n-1}\right)$ независимь, где

$$
y(t)=\omega^{-1} x(t)+\int_{0}^{t} x(\tau) d \tau, \quad 0 \leqslant t \leqslant \beta .
$$


Случайный процесс $\{y(t), 0 \leqslant t \leqslant \beta\}$ является гауссовым с нулевым средним и корреляционной функцией

$$
\begin{aligned}
M(y(t) y(s))= & \frac{1}{2 m \omega^{2} \operatorname{sh} \frac{\beta \omega}{2}}\left\{2\left[\omega^{-1}+\min (s, t)\right] \operatorname{sh} \frac{\beta \omega}{2}-\frac{1}{\omega} \operatorname{ch} \frac{\beta \omega}{2}+\right. \\
& +\frac{1}{\omega}\left[\operatorname{ch}\left(\omega s-\frac{\beta \omega}{2}\right)+\operatorname{sh}\left(\omega s-\frac{\beta \omega}{2}\right)\right]+ \\
& \left.+\frac{1}{\omega}\left[\operatorname{ch}\left(\omega t-\frac{\beta \omega}{2}\right)+\operatorname{sh}\left(\omega t-\frac{\beta \omega}{2}\right)\right]\right\} .
\end{aligned}
$$

Формула (27) позволяет установить, что гауссова случайная величина $G \equiv y(t)-y(s)$ распределена нормально с нулевым средним и дисперсией $(t-s) /\left(m \omega^{2}\right)$, где $t>s$, т.е.

$$
G \sim N\left(0, \frac{t-s}{m \omega^{2}}\right) .
$$

Следует отметить, что если рассматривать $x(t)$ как случайную функшию, то интеграл

$$
\int_{0}^{t} x(\tau) d \tau
$$

введенный в разделах 4 и 5 , является стохастическим интегралом, определяемым как предел в среднем по заданной мере соответствующих интегральных сумм. Интеграл (28) сушествует тогда и только тогда, когда сушествует среднее $M\left(y^{2}\right)$. Последнее условие для боголюбовской меры выполнено, что следует, в частности, из формулы (27).

В заключение этого раздела заметим, что поскольку

$$
\int_{X} d \mu_{\mathrm{5}}(x)\left(\frac{1}{\beta} \int_{0}^{\beta} x(t) d t\right)^{2}=\frac{1}{\beta^{2}} \int_{0}^{\beta} d t \int_{0}^{\beta} d \tau B(t-\tau)=\frac{1}{\beta m \omega^{2}},
$$

то

$$
\lim _{\beta \rightarrow \infty} M\left(\frac{1}{\beta} \int_{0}^{\beta} x(t) d t\right)^{2}=0 .
$$

Так как боголюбовский случайный процесс имеет нулевое математическое ожидание $m \equiv M x(t)=0$, то можно говорить об эргодичности данного процесса в том смысле, что "временни́е" (по $\beta$ ) средние сходятся в среднем квадратичном к “фазовым" средним.

\section{6. МЕРА БОГОЛЮБОВА И ДИФФЕРЕНЦИАЛЬНЫЕ УРАВНЕНИЯ}

Для произвольной функции $x(t) \in X$, произвольного действительного числа $\xi$ и положительного числа $\beta$ определим функцию

$$
\delta_{\beta, \xi}(x)=\frac{1}{2 \pi} \int_{-\infty}^{\infty} d z e^{i z[y(\beta)-y(0)-\xi]},
$$

где функция $y(t), 0 \leqslant t \leqslant \beta$, задана соотношением (26). Функция (29) является аналогом функции Донскера-Лионса [12], введенной в свое время для исследования винеровской меры. 
Лемма. Математическое ожидание функиии (29) задается формулой

$$
E_{\mu_{\mathrm{B}}}\left\{\delta_{\beta, \xi}(x)\right\}=\sqrt{\frac{m \omega^{2}}{2 \pi \beta}} \exp \left(-\frac{m \omega^{2}}{2 \beta} \xi^{2}\right) .
$$

ДокАЗАтЕльство. Рассмотрим следующее математическое ожидание:

$$
\begin{aligned}
E_{\mu_{\mathrm{B}}}\left\{\delta_{\beta, \xi}(x)\right\} & =\int_{X} \delta_{\beta, \xi}(x) d \mu_{\mathrm{\textrm {B }}}(x)=\frac{1}{2 \pi} \int_{-\infty}^{\infty} d z e^{-i z \xi} \int_{X} d \mu_{\mathrm{Б}}(x) \exp \left[i z \int_{0}^{\beta} x(t) d t\right]= \\
& =\frac{1}{2 \pi} \int_{-\infty}^{\infty} d z e^{-i z \xi} \frac{1}{\sqrt{2 \pi a}} \int_{-\infty}^{\infty} e^{i z u} e^{-\frac{u^{2}}{2 a}} d u
\end{aligned}
$$

где

$$
a=\int_{0}^{\beta} \int_{0}^{\beta} B(t, s) d t d s=\frac{\beta}{m \omega^{2}} .
$$

Вычисляя интегралы в (31), получаем формулу (30). Лемма доказана.

Введем функцию

$$
u(\beta, \xi)=E_{\mu_{\mathrm{B}}}\left\{\delta_{\beta, \xi}(x) \exp \left(-\int_{0}^{\beta} V(y(s)-y(0)) d s\right)\right\}
$$

где действительная функция $V$ ограничена снизу.

ТЕОРЕМА 3. Функиия (32) является решением уравнения в частных производHblx

$$
\frac{\partial u}{\partial \beta}=\frac{1}{2 m \omega^{2}} \frac{\partial^{2} u}{\partial \xi^{2}}-V(\xi) u
$$

с начальным условием $u(0, \xi)=\delta(\xi)$ и граничными условиями $u(\beta, \pm \infty)=0$.

ДоКАЗАТЕЛЬСТво. Воспользуемся очевидной формулой

$$
\exp \left(-\int_{0}^{t} V(z(s)) d s\right)=1-\int_{0}^{t} V(z(\tau)) \exp \left(-\int_{0}^{\tau} V(z(s)) d s\right) d \tau
$$

Тогда

$$
\begin{aligned}
u(\beta, \xi)= & E_{\mu_{\mathrm{B}}}\left\{\delta_{\beta, \xi}(x)\right\}-\int_{0}^{\beta} E_{\mu_{\mathrm{B}}}\left\{\delta_{\beta, \xi}(x) V(y(\tau)-y(0)) \times\right. \\
& \left.\times \exp \left(-\int_{0}^{\tau} V(y(s)-y(0)) d s\right)\right\} d \tau= \\
= & E_{\mu_{\mathrm{B}}}\left\{\delta_{\beta, \xi}(x)\right\}-\frac{1}{2 \pi} \int_{0}^{\beta} d \tau \int_{-\infty}^{\infty} d z e^{-i z \xi} E_{\mu_{\mathrm{B}}}\{V(y(\tau)-y(0)) \times \\
& \left.\times \exp \left(-\int_{0}^{\tau} V(y(s)-y(0)) d s+i z(y(\beta)-y(0))\right)\right\} .
\end{aligned}
$$


Ho

$$
\begin{aligned}
E_{\mu_{\mathrm{B}}}\left\{V(y(\tau)-y(0)) \exp \left(-\int_{0}^{\tau} V(y(s)-y(0)) d s+i z(y(\beta)-y(0))\right)\right\}= \\
=E_{\mu_{\mathrm{S}}}\left\{\left[V(y(\tau)-y(0)) \exp \left(-\int_{0}^{\tau} V(y(s)-y(0)) d s+i z(y(\tau)-y(0))\right)\right] \times\right. \\
\quad \times[\exp (i z(y(\beta)-y(0))-i z(y(\tau)-y(0)))]\}= \\
=E_{\mu_{\mathrm{5}}}\left\{V(y(\tau)-y(0)) \exp \left(-\int_{0}^{\tau} V(y(s)-y(0)) d s+i z(y(\tau)-y(0))\right)\right\} \times \\
\quad \times E_{\mu_{\mathrm{B}}}\{\exp (i z(y(\beta)-y(\tau)))\}= \\
=\exp \left(-\frac{\beta-\tau}{2 m \omega^{2}} z^{2}\right) E_{\mu_{\mathrm{B}}}\{V(y(\tau)-y(0)) \times \\
\left.\quad \times \exp \left(-\int_{0}^{\tau} V(y(s)-y(0)) d s+i z(y(\tau)-y(0))\right)\right\}= \\
=\exp \left(-\frac{\beta-\tau}{2 m \omega^{2}} z^{2}\right) \int_{-\infty}^{\infty} d \eta V(\eta) e^{i z \eta} E_{\mu_{\mathrm{B}}}\left\{\exp \left(-\int_{0}^{\tau} V(y(s)-y(0)) d s\right) \delta_{\tau, \eta}(x)\right\},
\end{aligned}
$$

где были использованы теорема 2 и свойства функции (29). Таким образом, имеем

$$
\begin{aligned}
u(\beta, \xi)= & E_{\mu_{\mathrm{5}}}\left\{\delta_{\beta, \xi}(x)\right\}-\frac{1}{2 \pi} \int_{0}^{\beta} d \tau \int_{-\infty}^{\infty} d z e^{-i z \xi} \exp \left(-\frac{\beta-\tau}{2 m \omega^{2}} z^{2}\right) \times \\
& \times \int_{-\infty}^{\infty} d \eta V(\eta) e^{i z \eta} u(\tau, \eta) .
\end{aligned}
$$

Учитывая, что

$$
\int_{-\infty}^{\infty} d z \exp \left(-i z \xi-\frac{\beta-\tau}{2 m \omega^{2}} z^{2}+i z \eta\right)=\sqrt{\frac{2 \pi m \omega^{2}}{\beta-\tau}} \exp \left[-\frac{2 m \omega^{2}}{\beta-\tau} \frac{(\xi-\eta)^{2}}{4}\right],
$$

а также используя лемму, получаем

$$
\begin{aligned}
u(\beta, \xi)= & \sqrt{\frac{m \omega^{2}}{2 \pi \beta}} \exp \left(-\frac{m \omega^{2}}{2 \beta} \xi^{2}\right)- \\
& -\int_{0}^{\beta} \int_{-\infty}^{\infty} V(\eta) u(\tau, \eta) \sqrt{\frac{m \omega^{2}}{2 \pi(\beta-\tau)}} \exp \left[-\frac{m \omega^{2}}{2(\beta-\tau)}(\xi-\eta)^{2}\right] d \eta d \tau
\end{aligned}
$$

Непосредственной проверкой теперь нетрудно убедиться, что функция (34) удовлетворяет уравнению (33). Соответствуюшие начальное и граничные условия также, очевидно, выполнены. Теорема 3 доказана.

Благодарности. Автор благодарит профессора Дж. Джонсона за плодотворное обсуждение проблем функционального интегрирования (в частности, Дж. Джонсон указал на связь мер Винера и Боголюбова) и за любезно подаренную им прекрасную книгу [3], оказавшую сушественное влияние на содержание данной статьи. Автор благодарит А.И. Кириллова, прочитавшего рукопись статьи и сделавшего ряд важных замечаний.

Работа поддержана РФФИ, проект № 99-01-00887. 


\section{Список литературы}

[1] Н. Н. Боголюбов. ДАН СССР. 1954. Т. 99. С. 225.

[2] Д. П. Санкович. ТМФ. 1999. Т. 119. С. 345.

[3] G. W. Johnson, M. L. Lapidus. The Feynman Integral and Feynman's Operational Calculus. Oxford: Clarendon Press, 2000.

[4] H. L. Royden. Real Analysis. 3rd ed. NY: Macmillan, 1988.

[5] P. Lévy. Amer. J. Math. 1940. V. 62. P. 487.

[6] R. H. Cameron, W. T. Martin. Bull. Amer. Math. Soc. 1947. V. 53. P. 130.

[7] А. П. Прудников, Ю. А. Брычков, О. И. Маричев. Интегралы и ряды. Элементарные функции. М.: Наука, 1981.

[8] E. Хилле, Р. Филлипс. Функциональный анализ и полугруппы. М.: ИЛ, 1962.

[9] E. B. Davies. One-Parameter Semigroups. London: Academic Press, 1980.

[10] В. И. Богачев. Гауссовские меры. М.: Наука, 1997.

[11] Л. А. Янович. Приближенное вычисление континуальных интегралов по гауссовым мерам. Минск: Наука и техника, 1976.

[12] Х.-С. Го. Гауссовские меры в банаховых пространствах. М.: Мир, 1979.

Поступила в редакцию 16.XI.2000 г. 